J. Sustain. Wireless Syst.

Vol.02/ No. 1

Pages: 33- 41

http://irojournals.com/irosws/

DOI: https://doi.org/10.36548/jsws.2020.1.004

\title{
Interference Reduction Using Principles and Application of Cognitive Radar in Pulse Jammer Suppression
}

\author{
Dr. Robert Bestak \\ Department of Tele Communication Engineering \\ Czech Technical University in Prague \\ Prague, Czech Republic. \\ Email: robert.bestak@fel.cvut.cz
}

\begin{abstract}
The increase in the number of emitters operating at a frequency close to that of the modern radar systems impose a major challenge on the rapidly developing market for communication systems and the increasing demand for electromagnetic spectrum. Interference problems occur when multiple radar systems of the same type operate in the same environment with limited bandwidth availability and leads to frequency regions overlap. The radar systems incur significant performance losses due to the inferences caused under such circumstances. The concept of Cognitive Radar can provide solutions for such issues. This paper provides an improved Cognitive Radar architecture to address these challenges. The strong interference signals are suppressed using adaptive processing technique. The significant market advantages and application overview of this system is also presented in this paper.
\end{abstract}

Keywords: Cognitive radar; wireless communication; pulse jammer suppression; STFT; Blanking algorithm;

\section{Introduction}

The rapid growth of wireless communication systems and increasing demands emphasises on the significance of the limited resource called Spectral Bandwidth [1]. This implies that the probability of mutual interferences increases with the increase in the number of wireless communication systems. The performance figures of the radar systems that operate at closer frequencies of the bands utilized by these wireless communication systems may drop and be significantly affected such that it can no longer guarantee safe operation. In applications like air traffic control radars, the co-existence of similar radar systems is challenging as the operation frequency of these systems vary only by a few MHz. The effects of earth curvature or shadowing can affect the radar networks that make use of multiple radars to cover large areas simultaneously where a single radar system is not reachable.

It is not possible to guarantee an adequate offset frequency among the transmit frequencies of these discrete radar systems. The mutual interference of these systems are excluded. The concept of Cognitive Radar can be used to counteract such issues. Figure 1 presents a summary of the effects and the counteractive measure using the Cognitive Radar approach [2]. The radar is allowed to use its own cognitive abilities to independently perform an analysis and evaluation of the surrounding environment. The factors considered for the evaluation purpose include the previously acquired knowledge, an analysis and the appropriate awareness of the applicable scenario. 
J. Sustain. Wireless Syst.

Vol.02/ No. 1

Pages: 33- 41

http://irojournals.com/irosws/

DOI: https://doi.org/10.36548/jsws.2020.1.004

The interference characteristics resulting from the analysis can be used for adopting and modifying the radar transmitter, receiver, and signal processing for achieving the radar's optimum interference resistance to such systems [3]. Hence cognitive abilities are used for accomplishing this task by developing concepts and methodologies. In the radar system, implementation of this concept is performed by primarily establishing a suitable system architecture.

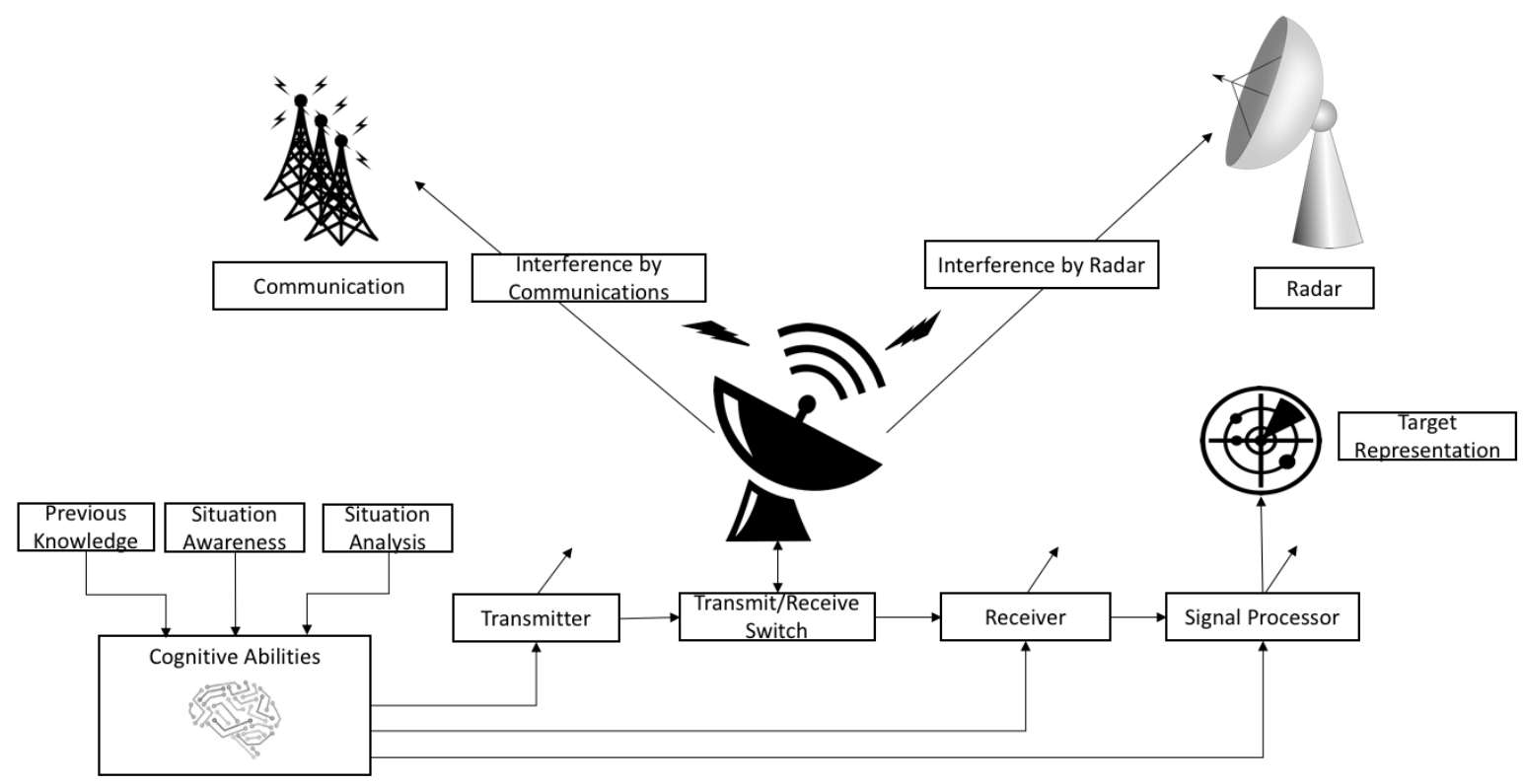

Figure 1 - Motivation for validation of the application of the Cognitive Radar with reference to interference

\section{Architecture of a Cognitive Radar System}

The architecture of a cognitive radar system is illustrated in figure 2. The required blocks are represented along with the communication between the blocks. The architecture can be broadly classified into four blocks for measurement of radar, processing and management of object, performance optimisation and radar resource management and task or mission control. The closed feedback loop is the major part of the concept. The fourth block, representing an additional element of architecture, enables the operator to actively influence the processes of the system. The individual blocks are further explained in this section. 
J. Sustain. Wireless Syst.

Vol.02/ No. 1

Pages: 33- 41

http://irojournals.com/irosws/

DOI: https://doi.org/10.36548/jsws.2020.1.004

\subsection{Radar Measurement}

Enabling the radar in detection, location or tracking of objects in the area for which it is responsible is termed as radar measurement which can be performed using several techniques of radar engineering. The major functions categorized into this block include signal generation, processing and tracking. The resolution and capability of observation of the radar are significantly influenced by factors such as transmitter signal generation and receiver signal processing. The undesired interference signals, detection and primary separation of target signals are affected by the processing of received signals. Noise and clutter signals such as weather, sea, ground and so on are categorised as interference signals. It also includes the interference from other emitters. Applying a Doppler processing technique that makes use of a coherent receiver and transmitter model is a common example of a simple clutter suppression system [4].

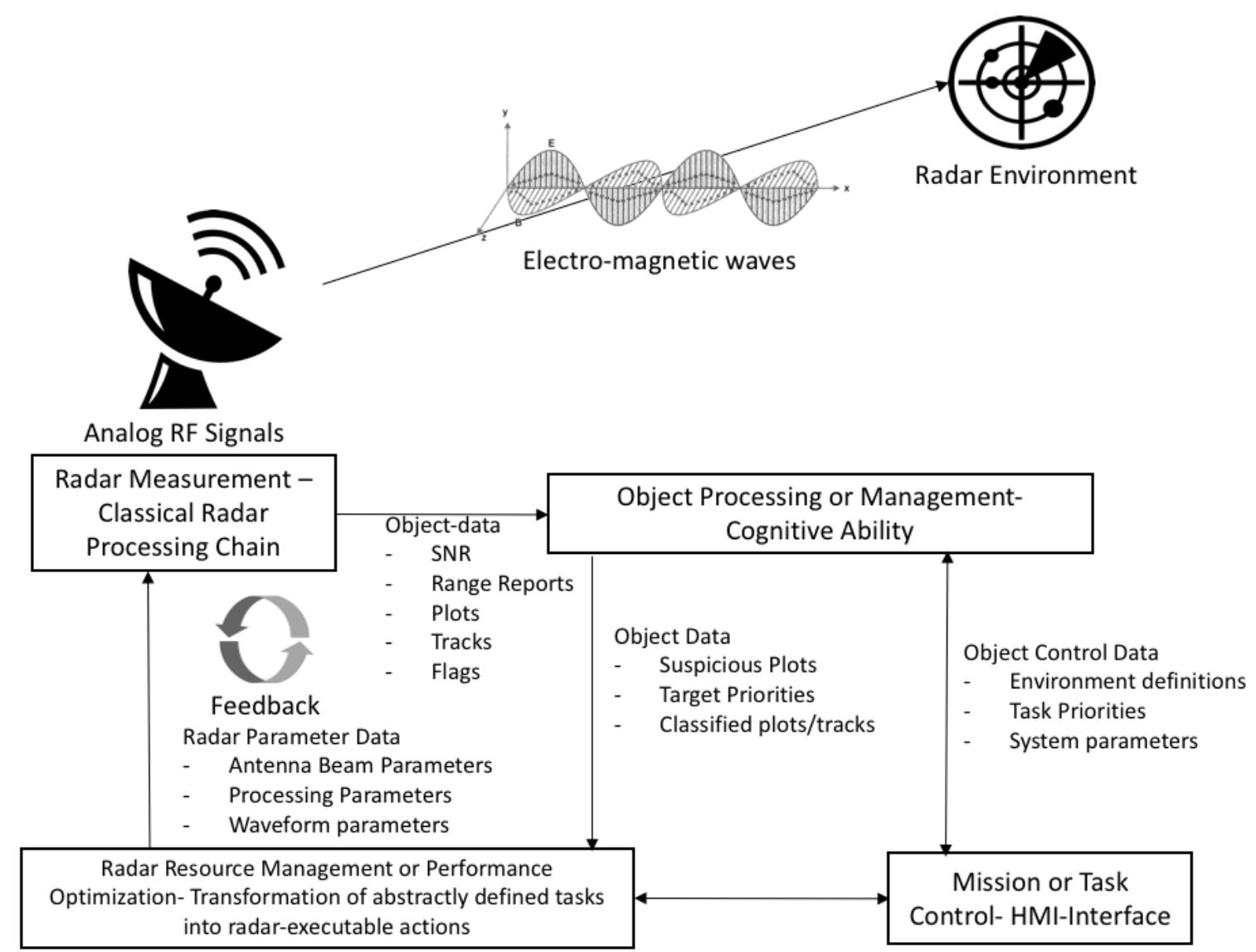

Figure 2 - Cognitive Radio Architecture 
J. Sustain. Wireless Syst.

Vol.02/ No. 1

Pages: 33- 41

http://irojournals.com/irosws/

DOI: https://doi.org/10.36548/jsws.2020.1.004

In order to ensure effective interference suppression, it is essential to involve more complex patter recognition techniques. Primarily, it is crucial to detect the appearance of an interference situation. This can be done by evaluating the measurement of the radar and use methods in parallel and similar to normal plot processing for matching it with the expected radar appearances. The obtained inferences in terms of tracks, plots, interference extensions or positions are transferred to the next block for further processing [5].

\subsection{Object Processing and Management}

The management and processing of object is performed by continuously processing the data generated by the radar measurement. Based on the current environment of the radar, an optimal detection performance is guaranteed by evaluation of the detected targets based on their significance and to establish appropriate measures [6]. This section can be classified into three steps namely target classification, situational awareness and target-related decision. It is possible for the radar system to automatically make accurate interpretation by evaluating the current environmental situation using the autonomous learning processes in this level and make appropriate decisions to solve the tasks optimally.

\subsection{Radar Resource Management and Performance Optimization}

At this level, the task that is defined abstractly is translated into activities that is to be executed by the radar system considering the predefined priorities of target. The major subgroups of this architectural component are performance and resource optimization. The technical realization of the abstract task description is performed by the performance optimization in an action executable by the radar system. In case of resource optimization, it is essential to develop appropriate time schedules or parameters that can determine the time and task that is to be executed at the specified system location. For autonomous execution of the task, the technical properties of the radar system are to be considered that specifies the available degrees of freedom to the radar system [7]. Selection of an appropriate waveform based on the factors like modulation, bandwidth, variation in transmission frequency and so on for the typical radar-technical degrees. The characteristics of the antenna design can also be customised.

\subsection{Mission or Task Control}

This block aids in performing the prioritization of external task. This assists in illuminator of individual locations by the operator at an increased frequency compared to others. Based on the availability of the required degrees of freedom in the radar, the radar modes like single target track or track while scan can also be triggered manually [8]. While tracking of several targets are performed, a 
J. Sustain. Wireless Syst.

Vol.02/ No. 1

Pages: 33- 41

http://irojournals.com/irosws/

DOI: https://doi.org/10.36548/jsws.2020.1.004

single target search can be ensured in the track while scan technique. The observation of a single track can be performed by focusing the entire energy of a single target track.

\section{Proposed Work}

Suppression of pulsed interferences is a potential application of cognitive radars. The requirement of wide coverage area for these networks make it a suitable choice. In this system, the network makes use of several radars operated simultaneously thereby causing overlaps in the coverage area [9]. This results in pulse interference between individual radar systems. Figure 4 represents a prospective scenario that consist of $\mathrm{N}$ interfering radars.

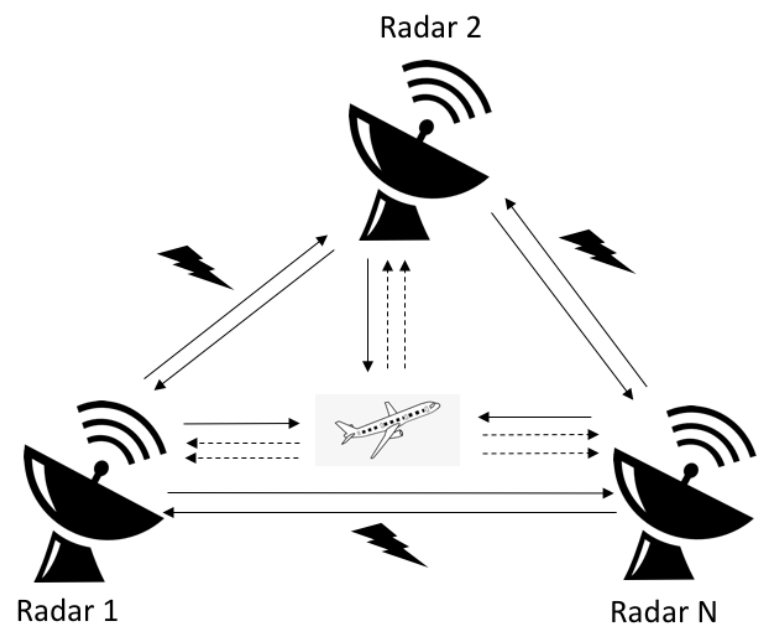

Figure 3- N radar system with interference in radar-to-radar communication

Among the $\mathrm{N}$ radars that are simultaneously operating, the signal received by the $(\mathrm{N}-1)^{\text {th }}$ radar is given by the following expression

$$
r_{n-1}(t)=\sum_{n=1}^{N} \alpha_{n, n-1}(t) \cdot s_{n}\left(t-\tau_{n, n-1}\right)+(n-1)_{n-1}(t)
$$

in which $r_{n-1}(t)$ represents the signal received at the $\mathrm{n}^{\text {th }}$ radar, $\mathrm{t}$ is the time, $\mathrm{N}$ is the total number of radars in the system, $(n-1)_{n-1}(t)$ is the noise signal at the $\mathrm{n}^{\text {th }}$ radar receiver, $\tau_{n, n-1}$ is the time delay of the signal transmitted from the $\mathrm{n}^{\text {th }}$ radar to (n-1), $\alpha_{n, n-1}$ is the losses on the line of sight of the $\mathrm{n}^{\text {th }}$ and $(\mathrm{n}-1)^{\text {th }}$ radar and $s_{n}$ represents the signal at $\mathrm{n}^{\text {th }}$ radar. 
J. Sustain. Wireless Syst.

Vol.02/ No. 1

Pages: 33- 41

http://irojournals.com/irosws/

DOI: https://doi.org/10.36548/jsws.2020.1.004

In order to remove the unnecessary radar signal components such as the echo signals of the same radar and other components from the opposing radars, Short Time Fourier Transform (STFT) blanking is used [10]. The processing steps of this technique is represented in the flowchart below.

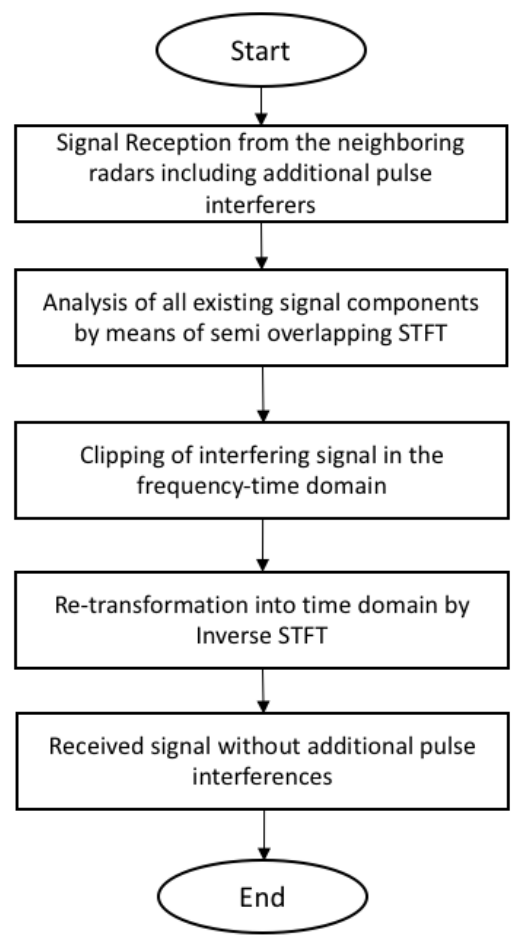

Figure 4- Flow chart of the STFT blanking technique for interference suppression

Additional pulse interference signals overlap the target signal that is received by the radar in the initial level. These overlap signals are most often in the range of the signals that are to be detected even though the presence is temporary. Based on the partial overlapping of the signals in the frequencytime domain, the existing components of the signal are to be analysed. A semi overlapping STFT can be used to achieve this in an efficient manner [11]. This helps in deriving the behaviour of the local frequency for every signal component with respect to time. Identification and removal of the interference signal is to be done in the next step. Simple and effective techniques for elimination can be applied on effective classification of interference signals.

\section{Results and Discussion}

A synthetic generated scenario is used for the demonstration of the result achieved on implementation of the STFT-blanking method. Real field tests may be used for verification of the 
J. Sustain. Wireless Syst.

Vol.02/ No. 1

Pages: 33- 41

http://irojournals.com/irosws/

DOI: https://doi.org/10.36548/jsws.2020.1.004

interference scenarios and their simulation. However, the synthetic data generated are consulted because of the flexibility of the configurations of various frequencies between the interfering signals and the target signals. In case of two signals received where one is the interference signal and other is the target signal, considering a test case where the bandwidth of both signals are $2.5 \mathrm{Mhz}$ and they are linear frequency modulated. The signals are received at a distance of $50 \mathrm{kms}$ at $330 \mu$ s time interval after emission of the radar signal. A positive chirp will be available in one signal which is the target signal in this scenario. The other signal or the interfering signal will contain a negative chirp as it occurs from the opposite radar. During the occurrence of a positive chirp, the frequency increases, and on the negative chirp, there is a decrease in the frequency. Signal to noise ratio of the target signal is $-10 \mathrm{~dB}$ and that of the interference signal is $+30 \mathrm{~dB}$ which is is another distinguishing feature of the system. The visibility of the interference signal increases when the target signal falls below the noise level.



Figure 5: Impact of STFS on audio signals

\section{Conclusion}

This paper illustrates the concept of cognitive radar that can be used for enhancing the existing radar framework with updates and improvements. Several radar prototypes such as sense and avoid, ground radars and navel radars use Hensoldt radars that make use of cognitive capabilities. The interference is considerably reduced in pule interferes on application of STFT blanking approach. The remainder is basically two individual interference pulses that are occurring at an interval of just one pulse length of the interference. The interference discontinuities are not eliminated completely and are characterised over the entire frequency domain. It is also possible to eliminate the pulses on subsequent treatment; however, it is not mandatory. The signal energy of the interference peak is smeared by the 
J. Sustain. Wireless Syst.

Vol.02/ No. 1

Pages: 33- 41

http://irojournals.com/irosws/

DOI: https://doi.org/10.36548/jsws.2020.1.004

pulse compression technique over a huge area in time domain. This makes most of the signal disappear under the noise level. The detection and classification of individual interference signals are performed with cognitive radar before selection of a suitable technique for successful elimination of interfering impulses.

\section{References}

[1] Steck, M., Neumann, C., \& Bockmair, M. (2018, June). Cognitive radar principles and application to interference reduction. In 2018 19th International Radar Symposium (IRS) (pp. 1-10). IEEE.

[2] Aubry, A., Carotenuto, V., De Maio, A., \& Govoni, M. A. (2018). Multi-snapshot spectrum sensing for cognitive radar via block-sparsity exploitation. IEEE Transactions on Signal Processing, 67(6), 1396-1406.

[3] Mishra, Kumar Vijay, and Yonina C. Eldar. "Performance of time delay estimation in a cognitive radar." In 2017 IEEE International Conference on Acoustics, Speech and Signal Processing (ICASSP), pp. 3141-3145. IEEE, 2017.

[4] Gui, R., Wang, W. Q., Pan, Y., \& Xu, J. (2018). Cognitive target tracking via angle-rangeDoppler estimation with transmit subaperturing FDA radar. IEEE Journal of Selected Topics in Signal Processing, 12(1), 76-89.

[5] Charlish, A., Hoffmann, F., Klemm, R., Nickel, U., \& Gierull, C. (2017). Cognitive radar management. Novel radar techniques and applications: waveform diversity and cognitive radar and target tracking and data fusion, 2, 157-193.

[6] Potapov, A. A., Zhang, W., \& Feng, T. (2018, August). Cognitive Radar in Fractal-Scaling Design. In 2018 International Conference on Sensing, Diagnostics, Prognostics, and Control (SDPC) (pp. 757-762). IEEE.

[7] Smith, G. E., Cammenga, Z., Mitchell, A., Bell, K. L., Johnson, J., Rangaswamy, M., \& Baker, C. (2016). Experiments with cognitive radar. IEEE Aerospace and Electronic Systems Magazine, 31(12), 34-46.

[8] Gurbuz, S. Z., Griffiths, H. D., Charlish, A., Rangaswamy, M., Greco, M. S., \& Bell, K. (2019). An Overview of Cognitive Radar: Past, Present, and Future. IEEE Aerospace and Electronic Systems Magazine, 34(12), 6-18.

[9] Brüggenwirth, S., Warnke, M., Wagner, S., \& Barth, K. (2019). Cognitive Radar for Classification. IEEE Aerospace and Electronic Systems Magazine, 34(12), 30-38.

[10] Bendory, T., Eldar, Y. C., \& Boumal, N. (2017). Non-convex phase retrieval from STFT measurements. IEEE Transactions on Information Theory, 64(1), 467-484.

[11] Zhang, Z., Li, X., \& Zhong, S. (2018, May). Efficiently Spectrum Utilization Method of Angle Sensitive Communication System Based on Cognitive Radio. In 2018 2nd IEEE Advanced Information Management, Communicates, Electronic and Automation Control Conference (IMCEC) (pp. 1797-1800). IEEE. 